\title{
Toward very high resolution imaging in ultrasound-modulated optical tomography of biological tissues
}

Sava Sakadžić, Sri-Rajasekhar Kothapalli, Lihong V. Wang

Sava Sakadžić, Sri-Rajasekhar Kothapalli, Lihong V. Wang, "Toward very high resolution imaging in ultrasound-modulated optical tomography of biological tissues," Proc. SPIE 6086, Photons Plus Ultrasound: Imaging and Sensing 2006: The Seventh Conference on Biomedical Thermoacoustics, Optoacoustics, and Acousto-optics, 608614 (6 March 2006); doi: $10.1117 / 12.645285$

SPIE. Event: SPIE BiOS, 2006, San Jose, California, United States 


\title{
Towards very high resolution imaging in ultrasound-modulated optical tomography of biological tissues
}

\author{
Sava Sakadžić, Sri-Rajasekhar Kothapalli and Lihong V. Wang \\ Optical Imaging Laboratory, Department of Biomedical Engineering, \\ Texas A\&M University, College Station, Texas 77843-3120
}

\begin{abstract}
We explored the possibility of applying very high ultrasound frequencies to achieve very high resolution in ultrasound-modulated optical tomography of soft biological tissues. The ultrasound-modulated coherent light that traversed the scattering biological tissue was detected by a long-cavity and a large etendue confocal FabryPerot interferometer. We used various focused ultrasound transducers of $15 \mathrm{MHz}, 30 \mathrm{MHz}$, and $50 \mathrm{MHz}$ to obtain two dimensional images of optically absorbing objects positioned at a few millimeters depth below the surface of both optically scattering phantoms and soft biological tissue samples. This technology is complementary to other imaging technologies, such as confocal microscopy and optical-coherence tomography, and has potential for broad biomedical applications.
\end{abstract}

Keywords: ultrasound-modulated optical tomography, multiply scattered light, microscopy

\section{INTRODUCTION}

Great effort has been expended recently on developing new imaging modalities based on the optical properties of soft biological tissues in the visible and near-infrared wavelength regions. The optical properties of biological tissues at these wavelengths are related to the molecular structure of the tissue, which offers potential for the detection of physiological functions and abnormalities.

Ultrasound-modulated optical tomography ${ }^{1,2}$ (UOT) is a hybrid technique that has been proposed to provide better resolution for the optical imaging of soft biological tissue by combining ultrasonic resolution and optical contrast. In UOT, focused ultrasound and optical radiation of high temporal coherence are simultaneously applied to soft biological tissues. Collective motions of the optical scatterers and periodic changes in the optical index of refraction are generated by ultrasound to produce temporal fluctuations in the intensity of the speckles that are formed by the multiple-scattered light. ${ }^{3-7}$ The ultrasound-modulated component of light carries information about the optical properties of tissue from the region of interaction between the optical and ultrasonic waves.

Efficient detection of ultrasound-modulated light is a challenge due to the diffused light propagation and the uncorrelated phases among individual optical speckles. Several schemes of detection ${ }^{2-4,8-17}$ have been explored. The parallel speckle detection with a CCD camera, ${ }^{9}$ a Fabry-Perot interferometer ${ }^{16}$ or a photo-refractive crystal ${ }^{17}$ in the detection part of the UOT system results in better signal-to-noise ratio (SNR) then that based on a single square-law detector. To obtain resolution along the ultrasonic axis, several research groups have explored various techniques, including the ultrasound frequency sweep, ${ }^{8}$ computer tomography, ${ }^{18}$ and tracking of ultrasound pulses or short bursts by using different detectors. ${ }^{12,14,16,17}$ The pulsed ultrasound approaches provide direct resolution along the ultrasonic axis and are more compatible with conventional ultrasound imaging. Pulsed ultrasound has the capability of much higher instantaneous power than continuous-wave $(\mathrm{CW})$ ultrasound, reducing the undesired effects of increased noise due to its wide bandwidth. However, the current speed of CCD cameras is not sufficient for the real time recording of the ultrasound-modulated light intensity produced by the ultrasonic pulses. At present, the detection schemes based on the Fabry-Perot interferometer or the photo-refractive crystal are the most suitable choices for fast parallel speckle detection.

In this proceeding, we report on using various focused ultrasound transducers $(15 \mathrm{MHz}, 30 \mathrm{MHz}$, and 50 $\mathrm{MHz}$ ) to image optically absorbing objects positioned a few millimeters below the surface of both biological tissue-mimicking phantoms and soft biological tissue samples. In the detection part of our UOT system, we use a long-cavity scanning confocal Fabry-Perot interferometer (CFPI) that has a greater etendue than most CCD

Photons Plus Ultrasound: Imaging and Sensing 2006: The 7th Conference on Biomedical

Thermoacoustics, Optoacoustics, and Acousto-optics, edited by A. A. Oraevsky, et. al

Proc. of SPIE Vol. 6086, 608614 (2006) • 1605-7422/06/\$15・doi: 10.1117/12.645285

Proc. of SPIE Vol. 6086 608614-1 
cameras and provides parallel speckle processing. In addition, the CFPI can detect the propagation of highfrequency ultrasound pulses in real time while tolerating speckle decorrelation. Compared to the other approaches for the detection of UOT signals, the CFPI is especially efficient at detecting high ultrasound frequencies, where the background light can be filtered out effectively and the ultrasound-modulated component is being transmitted.

\section{EXPERIMENTAL SETUP}

The details of the experimental setup used to detect the UOT signals were described earlier. ${ }^{16}$ Figure 1 shows the configuration of the sample which is imaged in the experiments. The sample was gently pressed through a slit along the $\mathrm{Z}$ axis to create a semi-cylindrical bump. The orthogonal ultrasonic and optical beams [Fig. 1(a)] were focused to the same spot below the sample surface. Diffusely transmitted light was collected by an optical fiber with a $600-\mu \mathrm{m}$ core diameter. This configuration minimized the contribution of the unmodulated light from the shallow regions to the background and, in addition, enhanced the interaction between the ultrasound and some quasi-ballistic light that still existed at small imaging depths (up to one optical transport mean free path). Figure 1(b) presents the sample holder with the optically scattering sample. An optically absorbing object with the shape of the letter $\mathrm{C}$ is placed below the sample surface along the $\mathrm{Z}$ axis of the cylindrical bump. The axes of propagation of the ultrasonic and optical beams are denoted with $X$ and $Y$, respectively. In Fig. 1(b), an artificial cut is created in the scattering sample along the $Z$ axis in order to reveal the details of the object positioning.

In the experiment, the laser light (Coherent, Verdi; 532-nm wavelength) was focused onto a spot of $\sim 100 \mu \mathrm{m}$ in diameter below the surface of an otherwise scattering-free sample. The optical power delivered to the sample was between $30 \mathrm{~mW}$ and $300 \mathrm{~mW}$, depending on the type of sample. Although the $\mathrm{CW}$ power in this proofof-principle experiment exceeded the safety limit for average power, the duration of the light exposure to the sample can be reduced to only a few $\mu$ s for each ultrasound pulse propagation through the region of interest, and, therefore, the safety limit will not be exceeded in practice, even if the focus is maintained in a scattering medium. The sample was mounted on a three-axis $\left(X_{1}, Y_{1}\right.$, and $\left.Z_{1}\right)$ translational stage. The ultrasound transducer and the sample were immersed in water for acoustic coupling. The light focusing optics and the collecting fiber were also immersed in the same water tank. The collected light was coupled into the CFPI that was operated in transmission mode (50-cm cavity length, $0.1-\mathrm{mm}^{2}$ sr etendue, and $\sim 20$ finesse). The light sampled by the beam splitter was used in a cavity tuning procedure. The cavity was first swept through one free spectral range to find the position of the central frequency of the unmodulated light. Then, one CFPI mirror was displaced by a calibrated amount such that the cavity was tuned to the frequency of one sideband of the ultrasound-modulated light (15 MHz, $30 \mathrm{MHz}$, or $50 \mathrm{MHz}$ greater than the laser light frequency). An avalanche photo-diode (APD)
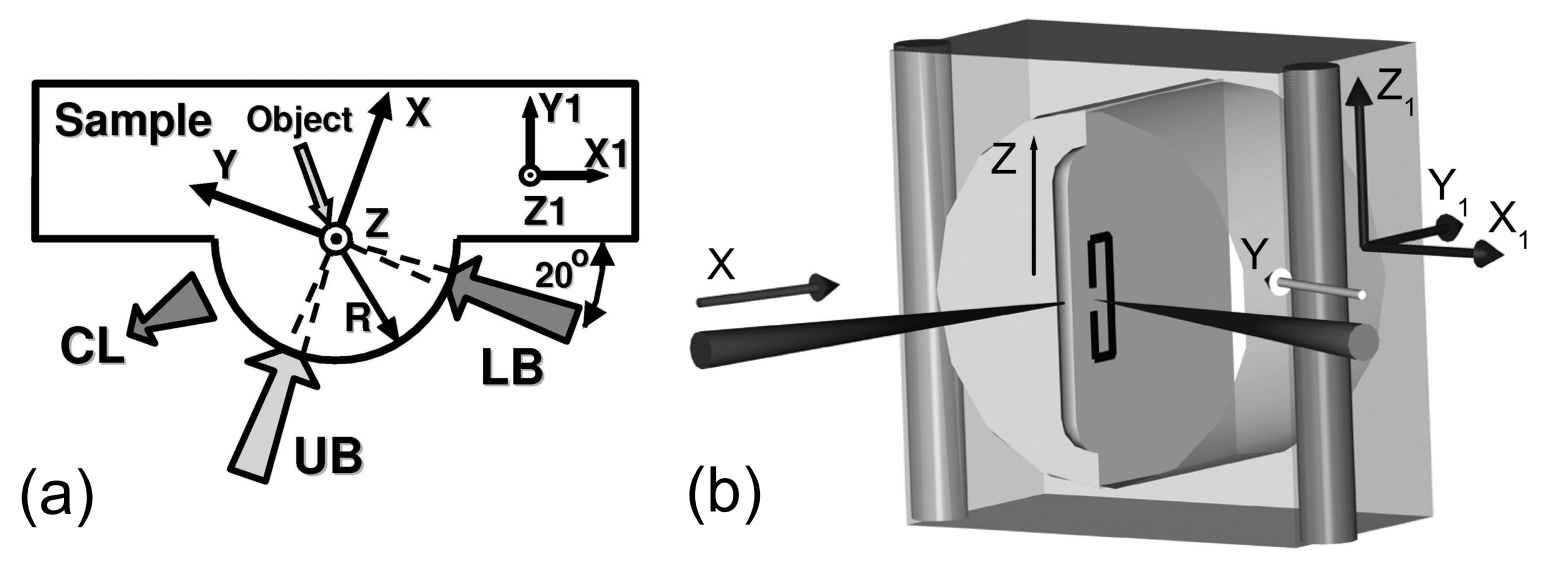

Figure 1. (a) Top view of the sample: UB, ultrasound beam; LB, incident light beam; CL, collected light; R, radius. (b) Schematic of the sample holder with the optically scattering sample: $X$, propagation direction of the ultrasound pulse; $Y$, propagation direction of light; $Z$, direction along which the sample was scanned in order to obtain two-dimensional image of the object; $\left(X_{1}, Y_{1}, Z_{1}\right)$, axes of the translational stage connected to the sample holder. 
(Advanced Photonix) acquired the light filtered by the interferometer, and the signal was sampled at $200 \mathrm{Ms} / \mathrm{sec}$ with a data acquisition board (GAGE, CS14200). A computer program written in LabView controlled the movement of the CFPI mirror and the other sequences of the control signals.

A trigger generator (Stanford Research, DG535) triggered both the ultrasound pulse generation and the data acquisition from the APD. Since the resonant frequency of the CFPI cavity coincided with one sideband of the ultrasound-modulated light, the signal acquired by the APD during the ultrasound propagation through the sample represented the distribution of the ultrasound-modulated optical intensity along the ultrasonic axis and, therefore, yielded a one-dimensional (1D) image along the $X$ direction. In each operational cycle, the resonant frequency of the CFPI was tuned first, and then data from 4000 ultrasound pulses were acquired in one second. Averaging over 10 to 50 cycles was usually necessary to obtain a satisfactory SNR for each 1D image. Two-dimensional images were obtained by scanning the sample along the $Z$ direction and acquiring each corresponding 1D image.

\section{RESULTS}

In the experiment, we used three ultrasound transducers with central frequencies of $15 \mathrm{MHz}, 30 \mathrm{MHz}$, and $50 \mathrm{MHz}$. The $15 \mathrm{MHz}$ transducer (Ultran, fused silica delay line, $4.7 \mathrm{~mm}$ lens diameter, $4.7 \mathrm{~mm}$ focal length, $15 \mathrm{MHz}$ estimated bandwidth) was driven by a pulser (GE Panametrics, 5072PR). The transducers with central frequencies of $30 \mathrm{MHz}$ and $50 \mathrm{MHz}$ (GE Panametrics, $4.25 \mu \mathrm{m}$ fused silica delay lines, $80 \%$ estimated fractional bandwidths, $6 \mathrm{~mm}$ element sizes, $5.5 \mathrm{~mm}$ focal lengths) were driven by square bipolar pulses with periods of $34 \mathrm{~ns}$ and 20 ns respectively. The pulses were generated by the trigger generator (Stanford Research, DG535) and amplified with an amplifier (Amplifier Research, 75A250). The ultrasound focal peak pressure measured by the needle hydrophone was between $2 \mathrm{MPa}$ and $4 \mathrm{MPa}$ for all three transducers, which is within the ultrasound safety limit at these frequencies for tissues without well-defined gas bodies. ${ }^{19}$

Figure 2 presents the value of the modulation depth (MD) as a function of the distance of the ultrasound focus from the surface of the chicken breast tissue sample. The MD is calculated as a ratio of the total measured ultrasound-modulated optical intensity to the unmodulated intensity. For all three transducers, the value of the MD is the highest at the surface of the sample $(\sim 1 \%)$, where focused beams of light and ultrasound interact as if in an optically clear medium. With increased depth of the ultrasound focus from the sample surface, the value of the MD decreases. A more rapid decay rate of the MD is observable at the higher ultrasound frequencies and close to the sample surface in the region where the light is not completely diffused. In general, the MD depends on both the optical and mechanical properties of the sample, as well as on the optical and ultrasonic parameters.

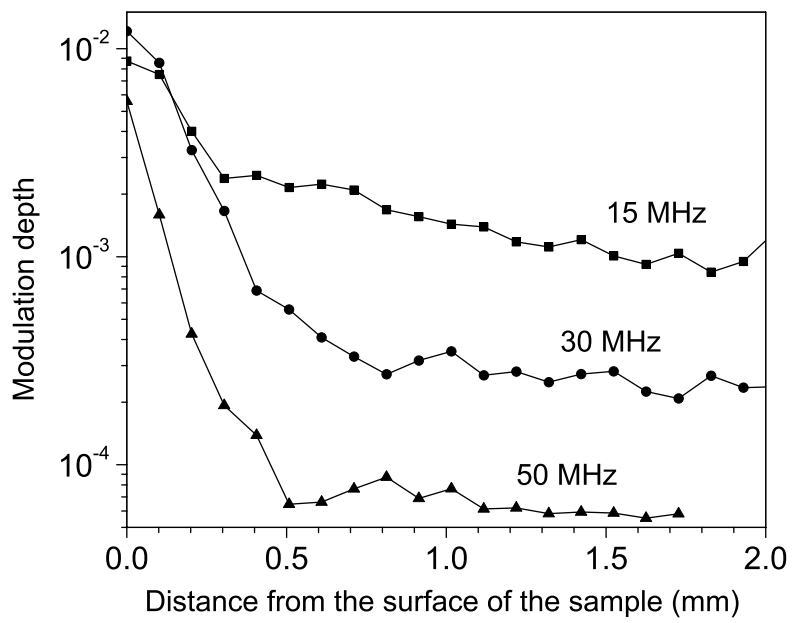

Figure 2. Modulation depth dependence on the distance of the ultrasound focus from the surface of the chicken breast tissue sample. The lines with solid squares, circles, and triangles correspond to the central frequencies of $15 \mathrm{MHz}, 30 \mathrm{MHz}$, and $50 \mathrm{MHz}$, respectively. 
When considering the ultrasound frequency, the frequency dependence of the ultrasound attenuation and the frequency dependence of the light modulation in the optically turbid medium (calculated within the framework of the diffusing-wave spectroscopy ${ }^{7}$ ) contribute to the lower MD values. Shorter and better focused ultrasound pulses at higher ultrasound frequencies occupy smaller volume, which also decreases the probability of interaction of the diffused light with ultrasound. However, by measuring the ultrasound-modulated optical intensity with the CFPI, the background of non-modulated light is suppressed up to one hundred times for the ultrasound central frequency of $50 \mathrm{MHz}$.

Figure 3 presents images of the same light absorbing object (placed in a tissue-mimicking phantom) obtained with the three previously described ultrasonic transducers having central frequencies of $15 \mathrm{MHz}, 30 \mathrm{MHz}$, and $50 \mathrm{MHz}$, respectively. The optically scattering sample was prepared from agar and intralipid (Lyposine 20\%), with a $1-\mathrm{mm}$ optical transport mean free path and with a $3.0-\mathrm{mm}$ radii of curvature in the cylindrical bump. The object with the shape of a letter C [Fig. 1(b)] was made from a 100- $\mu$ m thick black latex sheet, which was transparent for ultrasound but absorptive for light. It was placed in the center of the curvature of the prepared sample, i.e., $3.0 \mathrm{~mm}$ below the surface of the sample. The wide side of the object was parallel to the ultrasound beam and perpendicular to the light beam. We took the difference between the profiles of the modulated intensity along the $X$ axis and the typical profile without the object present. Subsequently, we divided the difference by the latter profile point-by-point to obtain the relative values, which are shown as a contour-plot image with five equally spaced levels between 0 and 1 [Fig. 3]. In all three cases, good resolution and very high contrast images of the object were obtained. In addition, the best SNR was obtained by using the ultrasound transducer with the central frequency of $30 \mathrm{MHz}$. There are two main reasons for the difference in SNR among the images presented in Fig. 3. The first reason is that temporal instabilities in the laser central frequency and in the position of the CFPI resonance have more influence on the measurements at lower ultrasound frequencies, where the resonance of the CFPI is closer to the peak of the nonmodulated light. Therefore, the measurements with the $30-\mathrm{MHz}$ and $50-\mathrm{MHz}$ ultrasonic transducers were less sensitive to instabilities in our UOT system than the measurements made with the $15-\mathrm{MHz}$ ultrasonic transducer. The second reason is that the ultrasound transducer with the $50-\mathrm{MHz}$ central frequency produced a lower modulation depth (Fig. 2) than the other two transducers, which contributed to the lower SNR in Fig. 3(c).

Finally, Fig. 4 presents a well-resolved and very high contrast two-dimensional image of the optically absorbing object in the chicken breast tissue sample. Measurement is performed with the ultrasonic transducer with a central frequency of $30-\mathrm{MHz}$. The sample was prepared with 2-mm radii of curvature in the cylindrical bump. The object with the shape of a letter C [Fig. 1(b)] was also made from a 100- $\mu$ m thick black latex sheet and
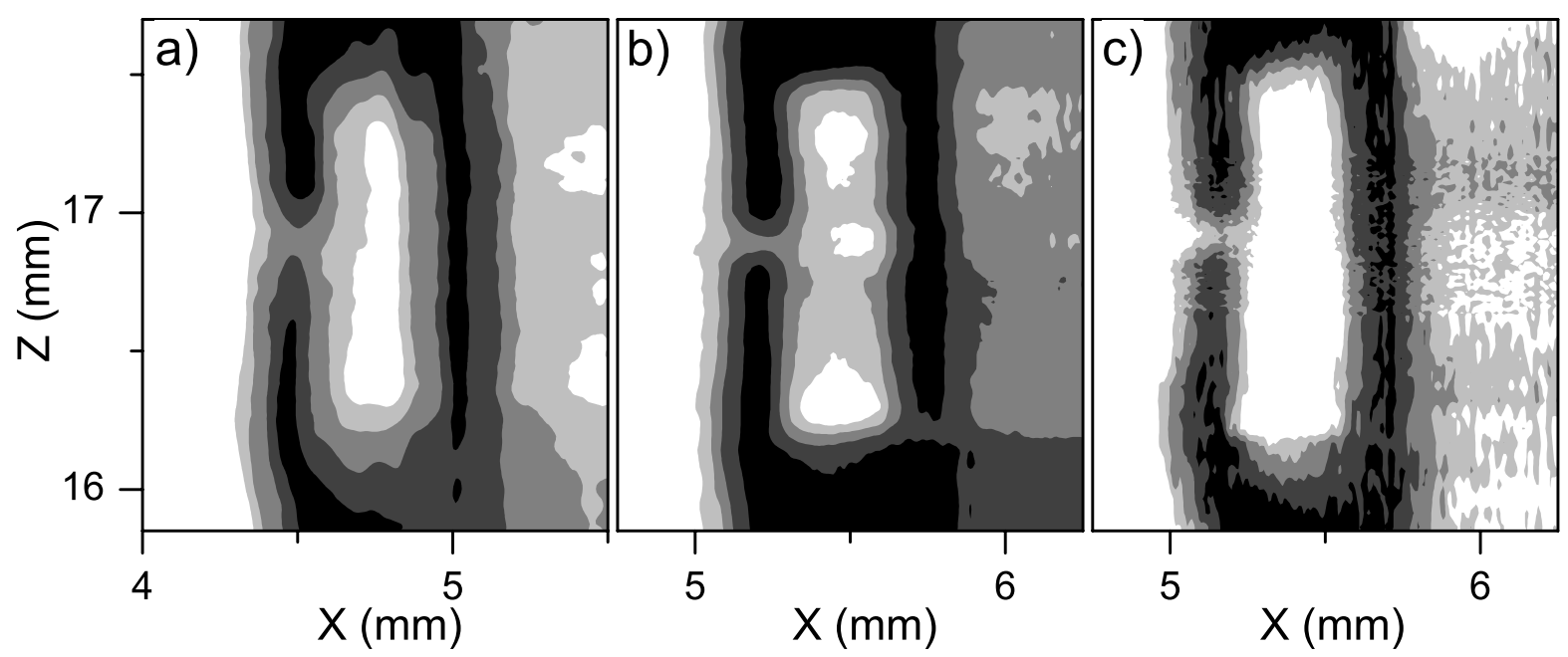

Figure 3. Images of an optically absorbing object placed $3.0 \mathrm{~mm}$ below the surface of the optically scattering tissue phantom. (a) Image of the object made with the $15-\mathrm{MHz}$ ultrasound transducer. (b) Image of the object made with the $30-\mathrm{MHz}$ ultrasound transducer. (c) Image of the object made with the 50-MHz ultrasound transducer. 


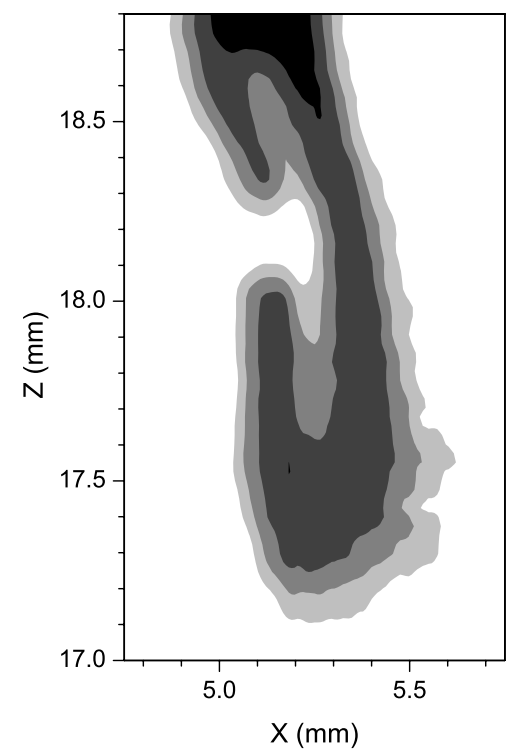

Figure 4. Two-dimensional image of an optically absorbing object placed $2.0 \mathrm{~mm}$ below the surface of a chicken breast tissue sample. Image is produced with the ultrasound transducer with a central frequency of $30 \mathrm{MHz}$.

placed in the center of the curvature of the prepared sample, $2 \mathrm{~mm}$ below the surface of the sample, with the wide side parallel to the ultrasound beam and perpendicular to the light beam. The relative profile of the measured modulated optical intensity is shown as a contour-plot image with five equally spaced levels between 0 and 1.

\section{CONCLUSION}

In summary, this study demonstrated the feasibility of using very high ultrasound frequencies $(30 \mathrm{MHz}$ and $50 \mathrm{MHz}$ ) to obtain high-resolution and high-contrast images with ultrasound-modulated optical tomography in biological tissues at an imaging depth of several millimeters. The resolution and the signal-to-noise ratio can be further improved by using better ultrasound sources and by optimizing the stability of the system. This technology can be readily integrated with conventional ultrasound imaging to provide complementary information.

This research was supported by the National Institute of Health grant R33 CA 094267. L. Wang's e-mail address is lwang@tamu.edu.

\section{REFERENCES}

1. F. A. Marks, H. W. Tomlinson, and G. W. Brooksby, "A comprehensive approach to brest cancer detection using light: photon localization by ultrasound modulation and tissue characterization by spectral discrimination," in Proc. SPIE, 1888, pp. 500-510, 1993.

2. L. V. Wang, S. L. Jacques, and X. Zhao, "Continuous-wave ultrasonic modulation of scattered laser light to image objects in turbid media," Opt. Lett. 20, pp. 629-631, 1995.

3. W. Leutz and G. Maret, "Ultrasonic modulation of multiply scattered-light," Physica B 204, pp. 14-19, 1995.

4. M. Kempe, M. Larionov, D. Zaslavsky, and A. Z. Genack, "Acousto-optic tomography with multiply scattered light," J. Opt. Soc. Am. A 14, pp. 1151-1158, 1997.

5. L. V. Wang, "Mechanisms of ultrasonic modulation of multiply scattered coherent light: an analytic model," Phys. Rev. Lett. 87, pp. 043903-(1-4), 2001.

6. S. Sakadzic and L. V. Wang, "Ultrasonic modulation of multiply scattered coherent light: an analytical model for anisotropically scattering media," Phys. Rev. E 66, pp. 026603-(19), 2002. 
7. S. Sakadzic and L. V. Wang, "Modulation of multiply scattered coherent light by ultrasonic pulses: An analytical model," Phys. Rev. E 72, pp. 036620-(1-12), 2005.

8. L. V. Wang and G. Ku, "Frequency-swept ultrasound-modulated optical tomography of scattering media," Opt. Lett. 23, pp. 975-977, 1998.

9. S. Leveque, A. C. Boccara, M. Lebec, and H. Saint-Jalmes, "Ultrasonic tagging of photon paths in scattering media: parallel speckle modulation processing," Opt. Lett. 24, pp. 181-183, 1999.

10. G. Yao, S.-L. Jiao, and L. V. Wang, "Frequency-swept ultrasound-modulated optical tomography in biological tissue by use of parallel detection," Opt. Lett. 25, pp. 734-736, 2000.

11. A. Lev, Z. Kotler, and B. G. Sfez, "Ultrasound tagged light imaging in turbid media in a reflectance geometry," Opt. Lett. 25, pp. 378-380, 2000.

12. M. Hisaka, T. Sugiura, and S. Kawata, "Optical cross-sectional imaging with pulse ultrasound wave assistance," J. Opt. Soc. Am. A-Opt. Image Sci. Vis. 18, pp. 1531-1534, 2001.

13. J. Li, G. Ku, and L. V. Wang, "Ultrasound-modulated optical tomography of biological tissue by use of contrast of laser speckles," Appl. Optics 41, pp. 6030-6035, 2002.

14. A. Lev and B. G. Sfez, "Pulsed ultrasound-modulated light tomography," Opt. Lett. 28, pp. 1549-1551, 2003.

15. M. Gross, P. Goy, and M. Al-Koussa, "Shot-noise detection of ultrasound-tagged photons in ultrasoundmodulated optical imaging," Opt. Lett. 28, pp. 2482-2484, 2003.

16. S. Sakadzic and L. V. Wang, "High-resolution ultrasound-modulated optical tomography in biological tissues," Opt. Lett 29, pp. 2770-2772, Dec 2004.

17. T. W. Murray, L. Sui, G. Maguluri, R. A. Roy, A. Nieva, F. Blonigen, and C. A. DiMarzio, "Detection of ultrasound modulated photons in diffuse media using the photorefractive effect," Opt. Lett. 29, pp. 2509$2511,2004$.

18. J. Li and L. V. Wang, "Ultrasound-modulated optical computed tomography of biological tissues," Appl. Phys. Lett 84, pp. 1597-1599, 2004.

19. AUIM, http://www.aium.org/, Mammalian In Vivo Ultrasonic Biological Effects, 1992. 